\title{
Frame-Based Random Access with Interference Cancellation across Frames for Massive Machine Type Communications
}

\author{
Minjoong Rim and Seungyeob Chae \\ Department of Information and Communication Engineering, Dongguk University, 30 Pildong-ro 1 Gil, Jung-gu, \\ Seoul 100-715, Republic of Korea
}

Correspondence should be addressed to Minjoong Rim; minjoong@dongguk.edu

Received 7 February 2017; Revised 25 April 2017; Accepted 15 June 2017; Published 19 July 2017

Academic Editor: Alessandro Bazzi

Copyright (C) 2017 Minjoong Rim and Seungyeob Chae. This is an open access article distributed under the Creative Commons Attribution License, which permits unrestricted use, distribution, and reproduction in any medium, provided the original work is properly cited.

\begin{abstract}
One of the main requirements for next generation mobile or wireless communication systems is to effectively support a large number of machine type communication devices for Internet of things applications. In order to improve the random access capability in frame-based slotted Aloha environments, coded random access techniques have been proposed, in which multiple copies of a packet are transmitted per frame and the copies are cancelled out from the received signal if any single copy is successfully received. They, however, may increase the transmission power by sending multiple copies per frame. Above all, for systems with a small number of slots per frame, they may not be able to improve the performance by readily reaching a congested state. This paper proposes a new frame-based random access scheme, which sends at most one copy of a packet per frame but uses interference cancellation to improve the performance. If a successfully received packet is a retransmitted one, the previously transmitted signals for the packet can be cancelled out from the received signals for trying to decode other received packets. The proposed scheme has different characteristics than coded random access schemes and can be also combined with them to further improve the performance.
\end{abstract}

\section{Introduction}

One of the major requirements for next generation wireless or cellular communication systems is to support a large number of machine type communication (MTC) devices in Internet of things (IoT) environments [1-4]. There are diverse types of IoT applications with different requirements, and in this paper we consider non-real-time services such as smart metering, surveillance, asset tracking, smart factory, and environmental monitoring, where uplink transmissions are dominant, the sizes of messages are generally short, the access delay is not critical, and devices require low power consumption to operate for years without maintenance [510]. As IoT services are widely used, the density of MTC devices will become very high and massive MTC techniques are required to support concurrent random accesses from a huge number of devices [5-10]. There are many works for efficiently supporting random accesses from a large number of devices, and recently a random access technique, called coded random access, has been proposed to improve the performance of frame-based slotted Aloha [11-16]. In a coded random access technique, multiple copies of a packet are transmitted over different slots in a frame and successive interference cancellation is performed starting with a successfully received copy, which can significantly reduce the collision probability. Coded random access techniques, however, may increase the waste in power consumption by transmitting multiple copies of a packet per frame and may not produce good results by readily reaching congested states if the number of slots per frame is not sufficiently large [17]. In this paper, we propose a random access scheme, which does not send multiple copies per frame but can improve the performance by successive interference cancellation of previously transmitted signals to decode other received packets. The proposed scheme has different characteristics than coded random access schemes and can be also combined with them to further improve the performance.

The rest of this paper is organized as follows. Section 2 describes conventional frame-based random access schemes 
including slotted Aloha and coded random access techniques. In Section 3, we propose a new random access scheme and present a combined scheme of the proposed and the coded random access. Simulation results are shown in Section 4 and conclusions are drawn in Section 5.

\section{Conventional Frame-Based Random Access}

2.1. Frame-Based Slotted Aloha. This paper considers framebased random access schemes, where each frame consists of $M$ slots with assuming that the length of a slot is the same as the maximum packet size. In a frame-based slotted Aloha scheme, a device with transmission data can randomly select a slot for packet transmission. For example, a slot position in a frame can be determined by a hash function or pseudorandom function generating an integer number from 0 to $M-$ 1 , written as

$$
\operatorname{slot}_{0}=\operatorname{Hash} 1\left(I D, S N, \text { frame }_{0}\right),
$$

where ID is the device identification (ID), $S N$ is the sequence number of the packet, and frame 0 is the frame number of the current frame considered. If a device transmitting a packet cannot receive acknowledgment (ACK) from the base station, it performs retransmission. We assume that the same data without modification is transmitted again for retransmission. Suppose that we could not receive ACK in the $n$th retransmission ( $n=0$ for the initial transmission). The difference between the next transmission frame frame $_{+1}$ (the $(n+1)$ th retransmission frame) and the current frame frame $_{0}$ (the $n$th retransmission frame), denoted as $d_{n}=$ frame $_{+1}-$ frame $_{0}$, can be randomly determined from $D_{\text {min }}$ to $D_{\min }+C W_{n}-1$, where $D_{\min }$ is the minimum distance between the two frames and $C W_{n}$ is the contention window size corresponding to retransmission number $n$. For example, the difference between the $(n+1)$ th retransmission frame and the $n$th retransmission frame can be determined by a hash function generating an integer number from $D_{\min }$ to $D_{\min }+C W_{n}-1$, expressed as

$$
d_{n}=\operatorname{Hash} 2(I D, S N, n), \quad(n=0, \ldots, N),
$$

where $N$ is the maximum number of $n$, in other words, the maximum number of retransmissions.

Figure 1 shows an example of frame-based slotted Aloha with $M=4$. In the first frame, there are collisions between the packets from Devices 1 and 2 and between the packets from Devices 3 and 4 . The packet from Device 2 is successfully decoded by the retransmission at the next frame and it requires more retransmissions for the packet from Device 1. In Figure 1, the rectangles with dotted lines show the successfully received packets possibly with retransmissions and the two packets numbered 1 and 2 are successfully received. The numbers show the order of successful receptions.

2.2. Coded Random Access with Repetition Coding. A framebased slotted Aloha scheme cannot achieve a good performance and a random access technique, called coded random access, has been proposed to improve the performance [1116]. There are various extensions of coded random access

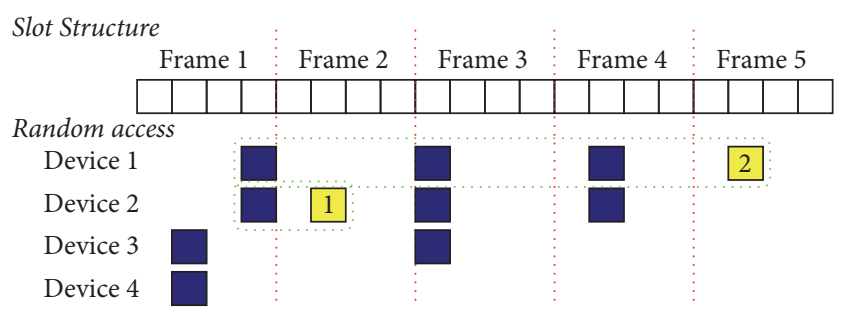

Successfully received without collision
Not successfully received

FIGURE 1: Slotted Aloha (the rectangles with dotted lines show the successfully received packets possibly with retransmissions and the two packets numbered 1 and 2 are successfully received).

schemes but in this paper we consider a basic form of coded random access, which uses repetition coding with code rate $1 / K$. In this scheme, a packet is duplicated to $K$ copies, and the $K$ copies are transmitted over $K$ randomly selected slots in a frame. For example, $K$ different slot positions can be determined by hash functions generating from 0 to $M-1$, written as

$$
\begin{aligned}
& \operatorname{slot}_{0, k}=\operatorname{Hash} 3\left(I D, S N, \text { frame }_{0}, k\right), \\
& \qquad(k=1, \ldots, K), \\
& \operatorname{slot}_{0, k 1} \neq \operatorname{slot}_{0, k 2}, \quad(k 1 \neq k 2) .
\end{aligned}
$$

If at least one copy of a packet is successfully received at a base station, then the device ID and the sequence number can be extracted from the packet and the slot positions for the other $K-1$ copies can be found using (3). Next, the packet is reconstructed and the channel value for each slot position is estimated using the correlation with the reconstructed packet. The $K-1$ copies are restored with the corresponding channel values and subtracted from the received signal. The base station tries to decode other packets on those slot positions and the same process is repeated until no more interference cancellation is feasible.

Figure 2 shows an example of the coded random access scheme with $K=2$, that is, code rate $1 / 2$. In the first frame, each of Devices 1,2,3, and 4 transmits two copies of a packet. There is no collision in the first slot of the first frame and hence the packet from Device 1 can be successfully decoded. Using the received packet from Device 1, the other copy in the fourth slot can be cancelled out from the received signal and the packet from Device 2 can be decoded. Further interference cancellation can be performed but no more successful decoding is feasible in this frame. In the figure, the five packets numbered from 1 to 5 are successfully decoded.

A coded random access scheme can produce satisfactory results if the number of slots per frame is large [1114]. However, it transmits multiple copies of a packet, and thus transmission power consumption may be increased compared to the conventional frame-based slotted Aloha scheme especially when the collision rate is small. Also, if the number of slots per frame is not sufficiently large, 

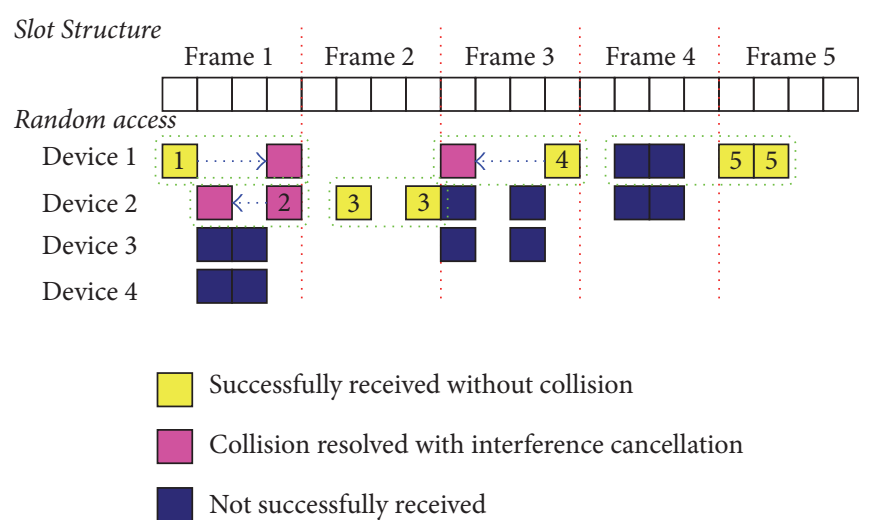

FIGURE 2: Coded random access with $K$ (the number of copies for a packet in a frame) $=2$ (the five packets numbered from 1 to 5 are successfully decoded).

transmitting multiple copies of a packet can readily result in congestion even with a small number of duplications and the performance improvement may be limited [17].

\section{Proposed Frame-Based Random Access}

3.1. Proposed Scheme. In this paper, we propose a new framebased random access scheme, in which a device sends at most one copy of a packet per frame without any overhead just the same as the frame-based slotted Aloha, but a base station performs successive interference cancellation similar to coded random access. If a successfully received packet at a base station is a retransmitted one, the base station performs interference cancellation of previously transmitted signals for trying to decode other previously received packets. Let $\widetilde{N}$ be the number of retransmissions considered for interference cancellation. If the number of slots per frame $(M)$ is sufficiently large, $\widetilde{N}$ can be simply set to the number of maximum retransmissions $(N)$. However, if $M$ is smaller than or equal to $N, \widetilde{N}$ is limited so that the information on the retransmission number $\tilde{n}$ can be extracted from the transmitted slot. Assuming

$$
\widetilde{N} \leq \min (M-1, N),
$$

the slot position can be selected similarly as (1), with the hash function

$$
\operatorname{slot}_{0}=\left(\operatorname{Hash} 1\left(I D, S N, \text { frame }_{0}\right)+\widetilde{n}\right) \bmod M,
$$

where mod is the modulo operation and

$$
\tilde{n}= \begin{cases}n & \text { if } n \leq \widetilde{N} \\ 0 & \text { otherwise }\end{cases}
$$

If a packet is successfully received at slot number slot, the device ID and the sequence number can be extracted from the packet and the retransmission number $\tilde{n}$ can be found using the following equation:

$$
\widetilde{n}=\left(\operatorname{slot}-\operatorname{Hash} 1\left(I D, S N, \text { frame }_{0}\right)\right) \bmod M .
$$

If $\tilde{n}$ is not zero, in other words, the packet is a retransmitted one; the previous frame for the packet can be found with

$$
\text { frame }_{-1}=\text { frame }_{0}-\operatorname{Hash} 2(I D, S N, \widetilde{n}-1)
$$

and the slot position in the frame frame $_{-1}$ can be known with

$$
\text { slot }_{-1}=\left(\operatorname{Hash} 1\left(I D, S N, \text { frame }_{-1}\right)+\tilde{n}-1\right) \bmod M .
$$

The base station performs the correlation with a reconstructed packet, finds the channel value on the slot, and subtracts the restored packet with the channel value from the received signal. Similarly, previous frame numbers frame $_{-1}, \ldots$, frame $_{-\tilde{n}}$ and slot positions on those frames slot $_{-1}, \ldots$, slot $_{-\tilde{n}}$ can be found with the following equations:

$$
\begin{array}{r}
\text { frame }_{-i}=\text { frame }_{0}-\sum_{j=1}^{i} \operatorname{Hash} 2(I D, S N, \tilde{n}-j), \\
(i=1, \ldots, \widetilde{n}), \\
\text { slot }_{-i}=\left(\operatorname{Hash} 1\left(I D, S N, \text { frame }_{-i}\right)+\tilde{n}-i\right) \bmod M, \\
(i=1, \ldots, \widetilde{n}) .
\end{array}
$$

Figure 3 shows an example of the proposed scheme. In the first frame, there are collisions between the packets from Devices 1 and 2 and between the packets from Devices 3 and 4 . In the second frame, Device 2 retransmits the packet and the transmission is successful. The successfully received packet is restored and the interference caused by the packet is cancelled out from the first frame, which results in the success of the packet from Device 1. In the third frame, a new packet is transmitted from Device 1 but cannot be decoded due to the collision with the packets from Devices 2 and 3. Device 1 performs retransmissions in the fourth and fifth frames and the success in the fifth frame can lead to successful decoding of other packets. In this figure, the six packets numbered from 1 to 6 are successfully decoded.

While multiple copies of a packet can be transmitted per frame and interference cancellation is performed within a frame in coded random access, at most one copy of a 


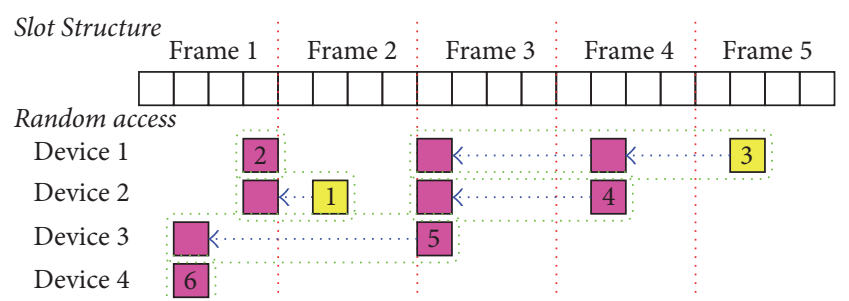

Successfully received without collision

Collision resolved with interference cancellation

FIGURE 3: Proposed random access scheme (the six packets numbered from 1 to 6 are successfully decoded.).

packet is transmitted in a frame but interference cancellation is performed across frames in the proposed scheme. The proposed scheme is the same as the conventional framebased slotted Aloha described in Section 2.1 if interference cancellation at a base station is not performed; in other words, $\widetilde{N}$ is zero. Hence, there is no transmission or computational overhead in the device side. On the other hand, in the base station side, there is some computational overhead for interference cancellation.

3.2. Combining the Proposed and Coded Random Access Schemes. If the number of slots per frame is large, a coded random access scheme can produce good results since there is a high probability of finding slots without collisions even with a large number of accesses, which can initiate the interference cancellation process. With a large number of slots per frame, the proposed scheme can be also combined with a coded random access scheme in order to further improve the performance. Let $K$ be the number of copies per packet and $\widetilde{N}$ be the number of retransmissions considered for interference cancellation satisfying the following equation:

$$
\widetilde{N} \leq \min \left(\frac{M}{K}-1, N\right) .
$$

Similar to (3) and (6), the $K$ slot positions can be calculated using

$$
\begin{array}{r}
\operatorname{slot}_{0, k}=\left(\operatorname{Hash}_{4}\left(I D, S N, \text { frame }_{0}, k\right)+\widetilde{n}\right) \bmod M, \\
(k=1, \ldots, K),
\end{array}
$$

where $\operatorname{Hash}_{4}(I D, S N$, frame f,$k)$ is a hash function generating $K$ slot positions at least $\widetilde{N}+1$ apart from one another, in other words, satisfying

$$
\begin{aligned}
\left|\operatorname{slot}_{0, k 1}-\operatorname{slot}_{0, k 2}+\alpha M\right|>\widetilde{N}, & \\
& (\alpha=-1,0,1 ; k 1 \neq k 2) .
\end{aligned}
$$

If one copy of a packet is successfully received at slot number slot, $\widetilde{n}$ can be found using the following equation:

$$
\begin{aligned}
\tilde{n}= & \min _{k=1, \ldots, K}\left(\left(\text { slot }- \text { Hash }\left(I D, S N, \text { frame }_{0}, k\right)\right)\right. \\
& \cdot \bmod M) .
\end{aligned}
$$

Based on this value, frame fri $_{-i}(i=1, \ldots, \widetilde{n})$ can be found using (11) and the slot position can be obtained with the following equation.

$$
\begin{aligned}
& \text { slot }_{-i, k} \\
& =\left(\operatorname{Hash} 4\left(I D, S N, \text { frame }_{-i}, k\right)+\widetilde{n}-i\right) \bmod M, \\
& \quad(k=1, \ldots, K ; i=1, \ldots, \widetilde{n}) .
\end{aligned}
$$

The interference cancellation process is repeated until no more packets can be successfully decoded.

If $\widetilde{N}$ is set to zero while $K>1$, the interference cancellation is performed only within a frame and this scheme becomes the coded random access scheme described in Section 2.2. If $\widetilde{N}>0$ and $K=1$, then this is the proposed scheme in Section 3.1, and if $\widetilde{N}=0$ and $K=1$, then this becomes the conventional slotted Aloha scheme in Section 2.1.

\section{Simulation Results}

In order to understand the characteristics of frame-based random access schemes described in Sections 2 and 3, we compare their collision probabilities and throughputs according to the number of devices concurrently performing random accesses. The considered schemes include the slotted Aloha described in Section 2.1, the coded random access schemes $(K=2,3$, or 4$)$ in Section 2.2, the proposed scheme in Section 3.1, and the combined scheme ( $K=2$ or 3 if (13) can be satisfied) in Section 3.2. In order to make the random access probability constant regardless of collision probabilities, the contention window size $C W_{n}$ is fixed to 7 for any $n$. Thus, with $D_{\min }=1$, a random number from 1 to 7 is generated to determine the position of the next transmission frame. The maximum number of retransmissions $N$ is set to 5 and the same number is used for the number of retransmissions considered for interference cancellation $\widetilde{N}$. Note that the number of transmissions is determined by the number of active devices regardless of schemes. Hence, the number of copies is just proportional to $K$ since each transmission is composed of $K$ copies. Coded random access schemes with $K$ repetitions transmits $K$ times more copies than the conventional frame-based slotted Aloha or the proposed scheme.

The collision probability is measured as the number of collision packets divided by the number of transmissions regardless of initial transmission or retransmissions. The throughput is measured as the number of successfully received packets divided by the total number of slots (the number of slots per frame $\times$ the number of frames) considered for simulation. Note that the maximum values of these two measurements are both 1 . We also assume that the interference cancellation is perfect although the remaining interference can degrade the performance in practice.

Figures 4 and 5 show the collision probabilities and the throughputs, respectively, with the number of slots per frame $M=16$. The combined scheme with $K=3$ is not considered since (13) is not satisfied with $K=3$. The coded random access schemes can produce good results compared 


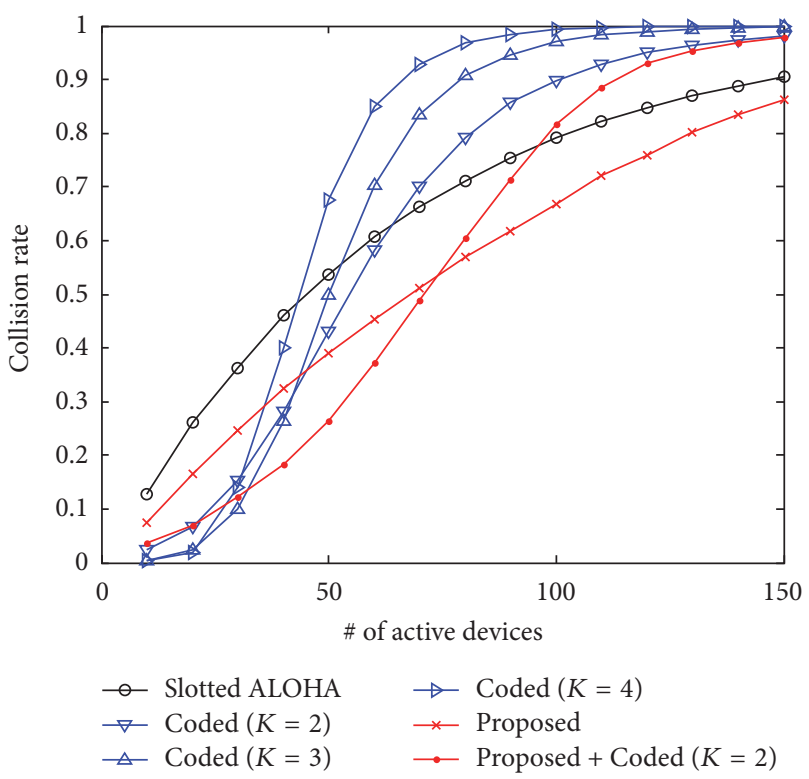

FIGURE 4: Collision probabilities with $M$ (the number of slots per frame) $=16$ (the collision probability is measured as the number of collision packets divided by the number of transmissions. The maximum is 1 ).

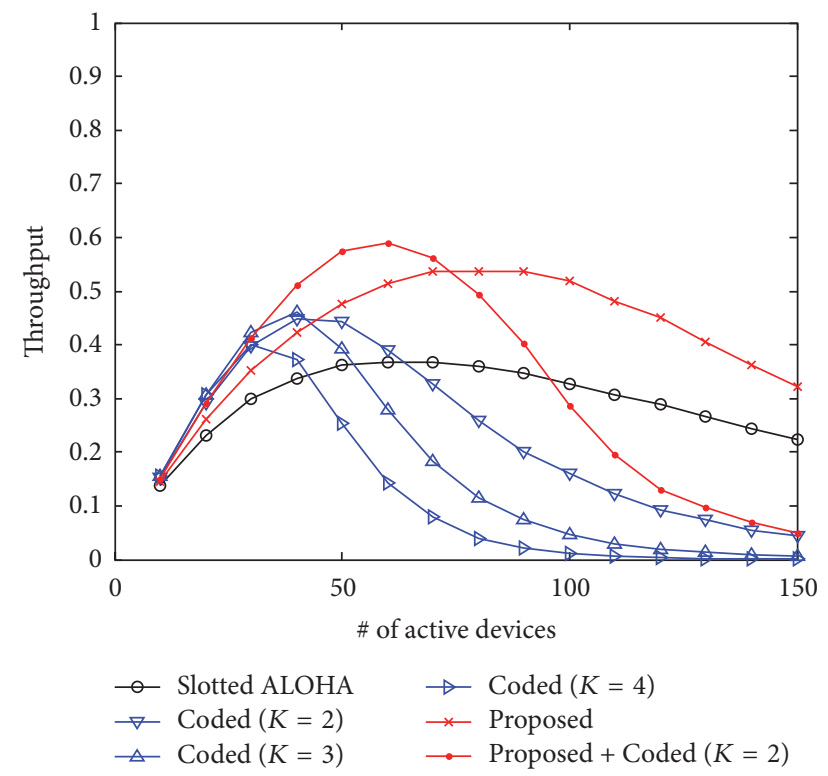

Figure 5: Throughputs with $M=16$ (the throughput is measured as the number of successfully received packets divided by the total number of slots considered for simulation. The maximum is 1 ).

to the conventional slotted Aloha but the improvement is not very significant since the number of slots per frame is not sufficiently large. The proposed scheme can produce satisfactory results and the combined scheme can achieve even better results.

If we increase the number of slots per frame, then the performance of coded random access schemes can be improved due to a high probability of having slots without

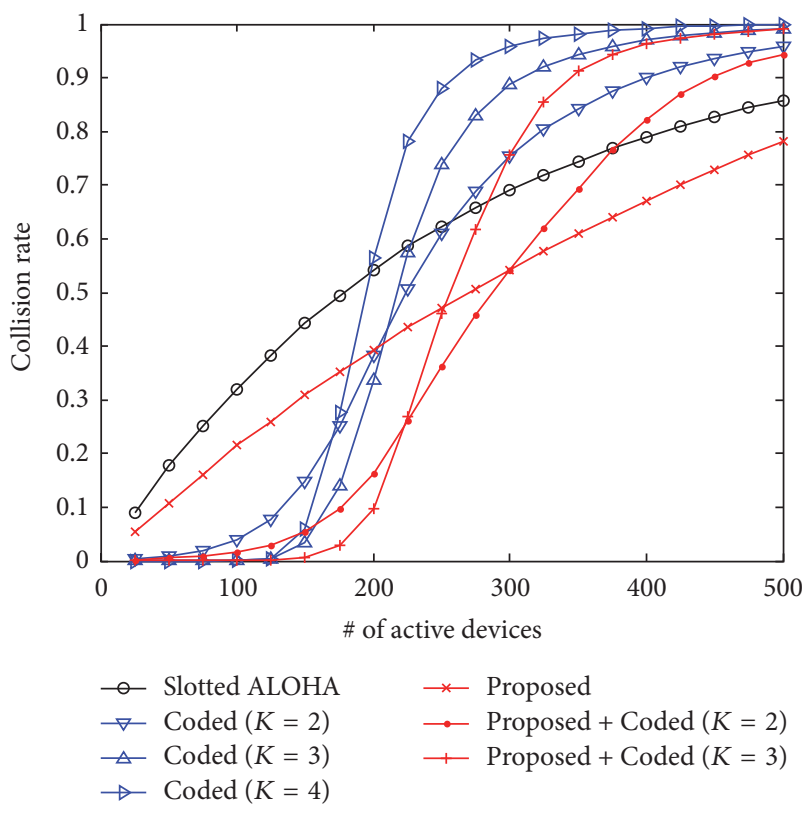

FIGURE 6: Collision probabilities with $M=64$.

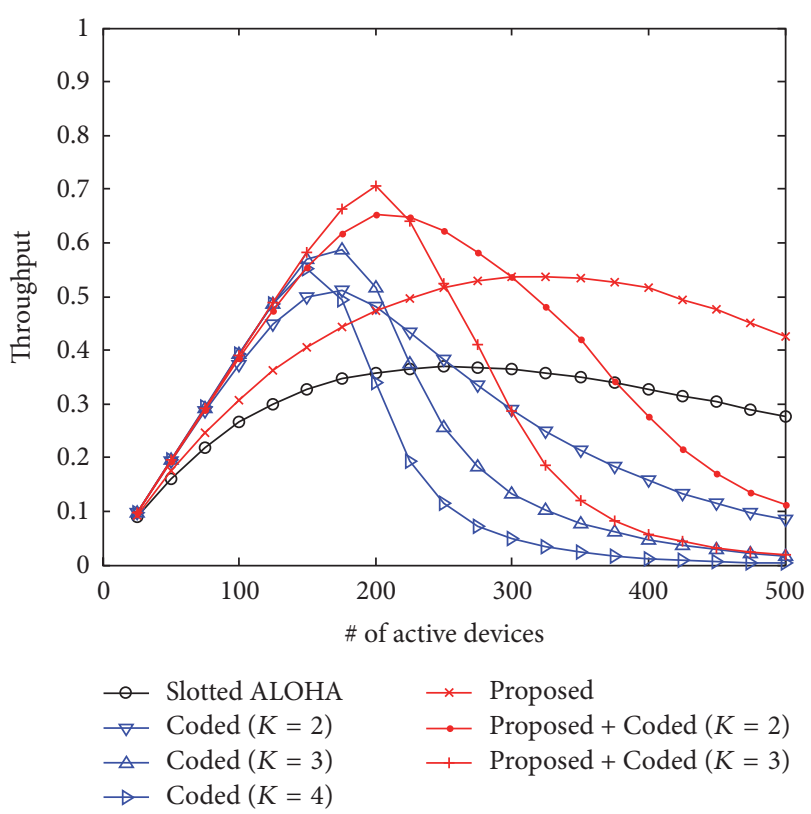

FIGURE 7: Throughputs with $M=64$.

collisions even with heavy random accesses, and successful receptions on those slots can initiate the interference cancellation process. Figures 6 and 7 show the cases with $M$ $=64$ and the performance improvements by coded random access schemes are considerable while the performance of the proposed is not noticeably affected by $M$. Coded random access schemes are very useful with a large number of slots per frame as long as increased transmission power consumption is acceptable. In such cases, the combined schemes with the proposed and the coded random access can produce even better results. 


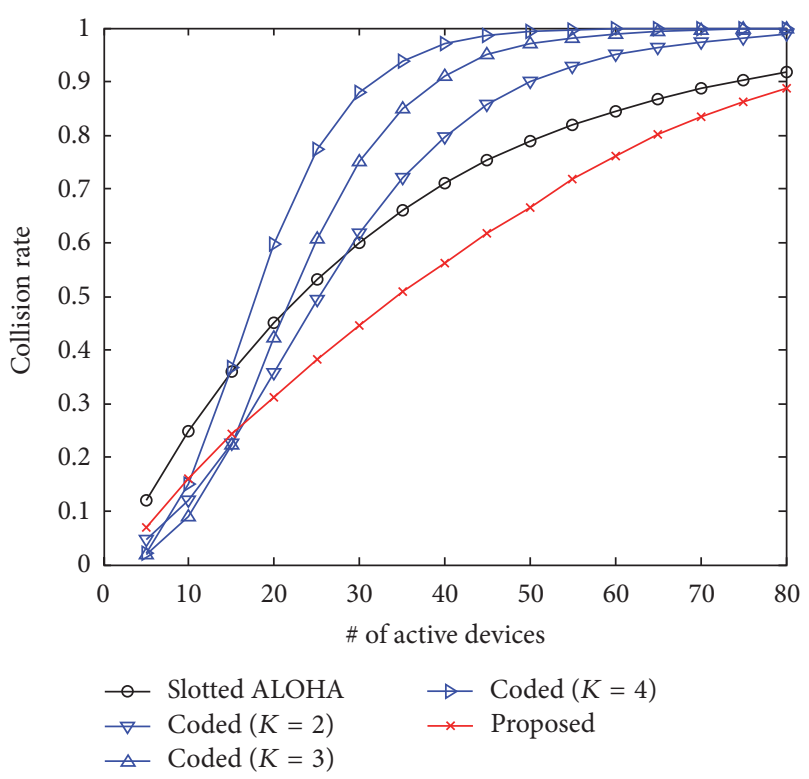

FIgURE 8: Collision probabilities with $M=8$.

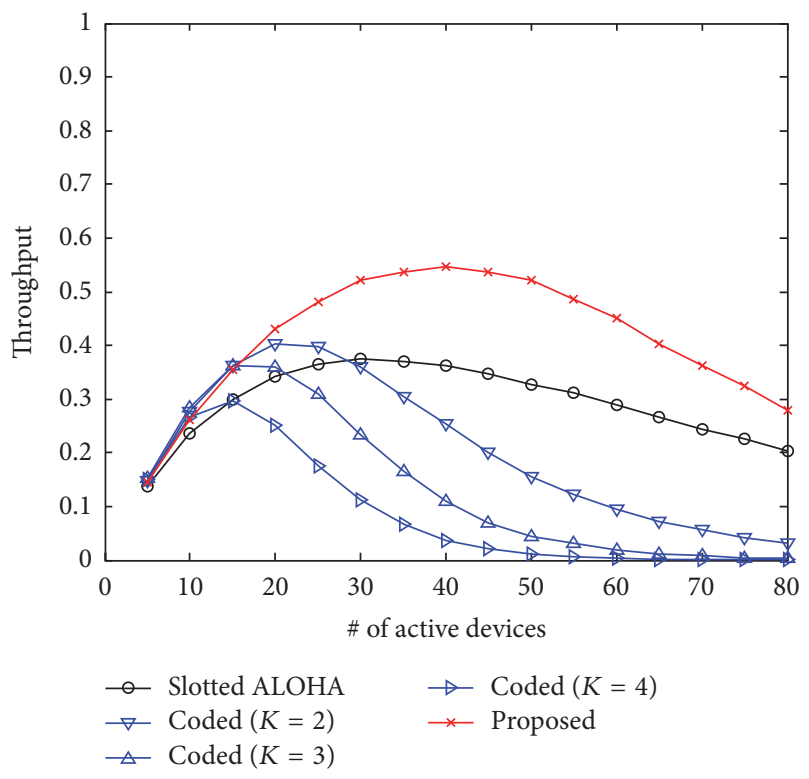

FIgURE 9: Throughputs with $M=8$.

Figures 8 and 9 show the results with a small number of slots per frame, more specifically $M=8$. In this case, the combined schemes are not considered since (13) is not satisfied even with $K=2$. The coded random access schemes hardly produce good results since it is not easy to find slots without collisions due to duplicated packets. On the other hand, the performance of the proposed scheme is not noticeably related to $M$ and it produces good results even with a small $M$.

\section{Conclusion}

In this paper, we have proposed a random access scheme to improve the performance of frame-based slotted Aloha.
The proposed scheme finds the frame and slot positions of the previous transmissions for a successfully received retransmitted packet and performs interference cancellation for trying to decode other received packets. While coded random access schemes may result in the waste of transmission power consumption by sending multiple copies of a packet per frame and the performance improvement may not be significant when the number of slots per frame is not large, the proposed scheme sends at most one copy of a packet per frame and can produce good results regardless of the number of slots per frame. The proposed scheme can be also combined with coded random access schemes and produce even better results especially when the number of slots per frame is large. In this paper, we assume that interference can be perfectly cancelled out from the received signal and in the future, more general environments and practical issues will be considered.

\section{Conflicts of Interest}

The authors declare that there are no conflicts of interest regarding the publication of this paper.

\section{Acknowledgments}

This work was supported by the National Research Foundation of Korea (NRF) grant funded by the Korea government (MSIP) (no. 2016R1A2B1008953).

\section{References}

[1] J. G. Andrews, S. Buzzi, and W. Choi, "What will 5G be?" IEEE Journal on Selected Areas in Communications, vol. 32, no. 6, pp. 1065-1082, 2014.

[2] M. Agiwal, A. Roy, and N. Saxena, "Next generation 5 G wireless networks: A comprehensive survey," IEEE Communications Surveys and Tutorials, vol. 18, no. 3, pp. 1617-1655, 2016.

[3] X. Li, J. B. Rao, and H. Zhang, "Engineering Machine-toMachine Traffic in 5G," IEEE Internet of Things Journal, vol. 3, no. 4, pp. 609-618, 2016.

[4] N. T. Le, M. A. Hossain, A. Islam, D. Kim, Y. Choi, and Y. M. Jang, "Survey of promising technologies for 5G networks," Mobile Information Systems, vol. 2016, Article ID 2676589, 25 pages, 2016.

[5] K. Zheng, S. Ou, J. Alonso-Zarate, M. Dohler, F. Liu, and H. Zhu, "Challenges of massive access in highly dense LTE-advanced networks with machine-to-machine communications," IEEE Wireless Communications, vol. 21, no. 3, pp. 12-18, 2014.

[6] M. R. Palattella, M. Dohler, A. Grieco et al., "Internet of things in the 5G Era: enablers, architecture, and business models," IEEE Journal on Selected Areas in Communications, vol. 34, no. 3, pp. 510-527, 2016.

[7] A. Ijaz, L. Zhang, M. Grau et al., "Enabling massive IoT in 5G and beyond systems: PHY radio frame design considerations," IEEE Access, vol. 4, pp. 3322-3339, 2016.

[8] Y. Yang, G. Song, W. Zhang, X. Ge, and C. Wang, "Neighboraware multiple access protocol for 5G mMTC applications," China Communications, vol. 13, no. Supplement2, pp. 80-88, 2016. 
[9] M. T. Islam, A.-E. M. Taha, and S. Akl, "A survey of access management techniques in machine type communications," IEEE Communications Magazine, vol. 52, no. 4, pp. 74-81, 2014.

[10] F. Yang and Q. Bi, "On novel access and scheduling schemes for IoT communications," Mobile Information Systems, vol. 2016, 2016.

[11] E. Paolini, Č. Stefanović, G. Liva, and P. Popovski, "Coded random access: Applying codes on graphs to design random access protocols," IEEE Communications Magazine, vol. 53, no. 6, pp. 144-150, 2015.

[12] Z. Zhang, C. Xu, and L. Ping, "Coded random access with distributed power control and multiple-packet reception," IEEE Wireless Communications Letters, vol. 4, no. 2, pp. 117-120, 2015.

[13] E. Paolini, G. Liva, and M. Chiani, "Coded slotted ALOHA: a graph-based method for uncoordinated multiple access," Institute of Electrical and Electronics Engineers. Transactions on Information Theory, vol. 61, no. 12, pp. 6815-6832, 2015.

[14] Č. Stefanović and P. Popovski, "ALOHA random access that operates as a rateless code," IEEE Transactions on Communications, vol. 61, no. 11, pp. 4653-4662, 2013.

[15] G. Wunder, C. Stefanovic, P. Popovski, and L. Thiele, "Compressive coded random access for massive MTC traffic in 5G systems," Asiloma Conference on Signals, Systems, and Computers, pp. 13-17, 2015.

[16] A. Purwita and K. Anwar, "Massive multiway relay networks applying coded random access," IEEE Transactions on Communications, vol. PP, no. 99, 2016.

[17] S. Chae, S. Cho, S. Kim, and M. Rim, "Coded random access with multiple coverage classes for massive machine type communication," in Proceedings of the 2016 International Conference on Information and Communication Technology Convergence (ICTC’16), pp. 882-886, Jeju, South Korea, October 2016. 

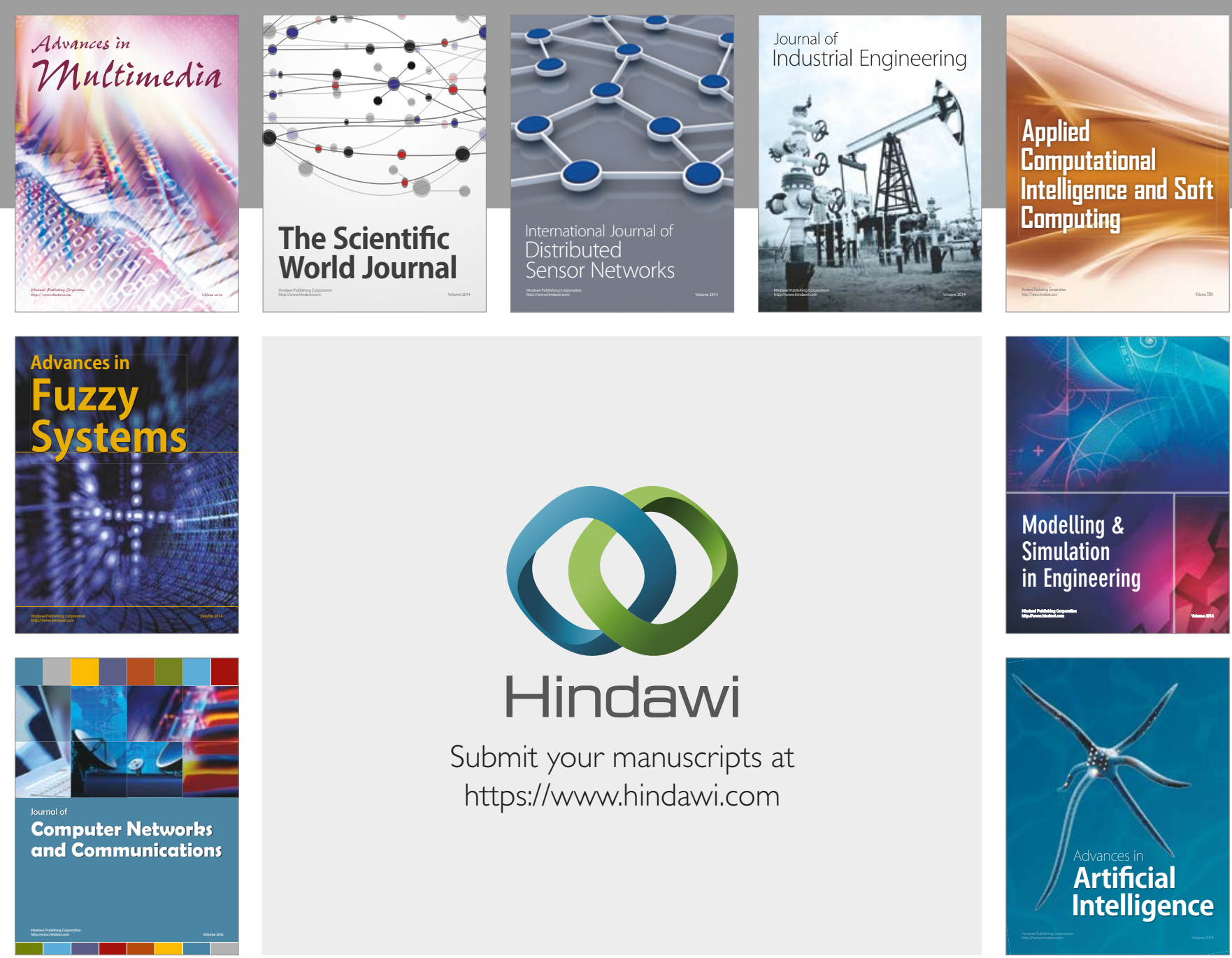

\section{Hindawi}

Submit your manuscripts at

https://www.hindawi.com
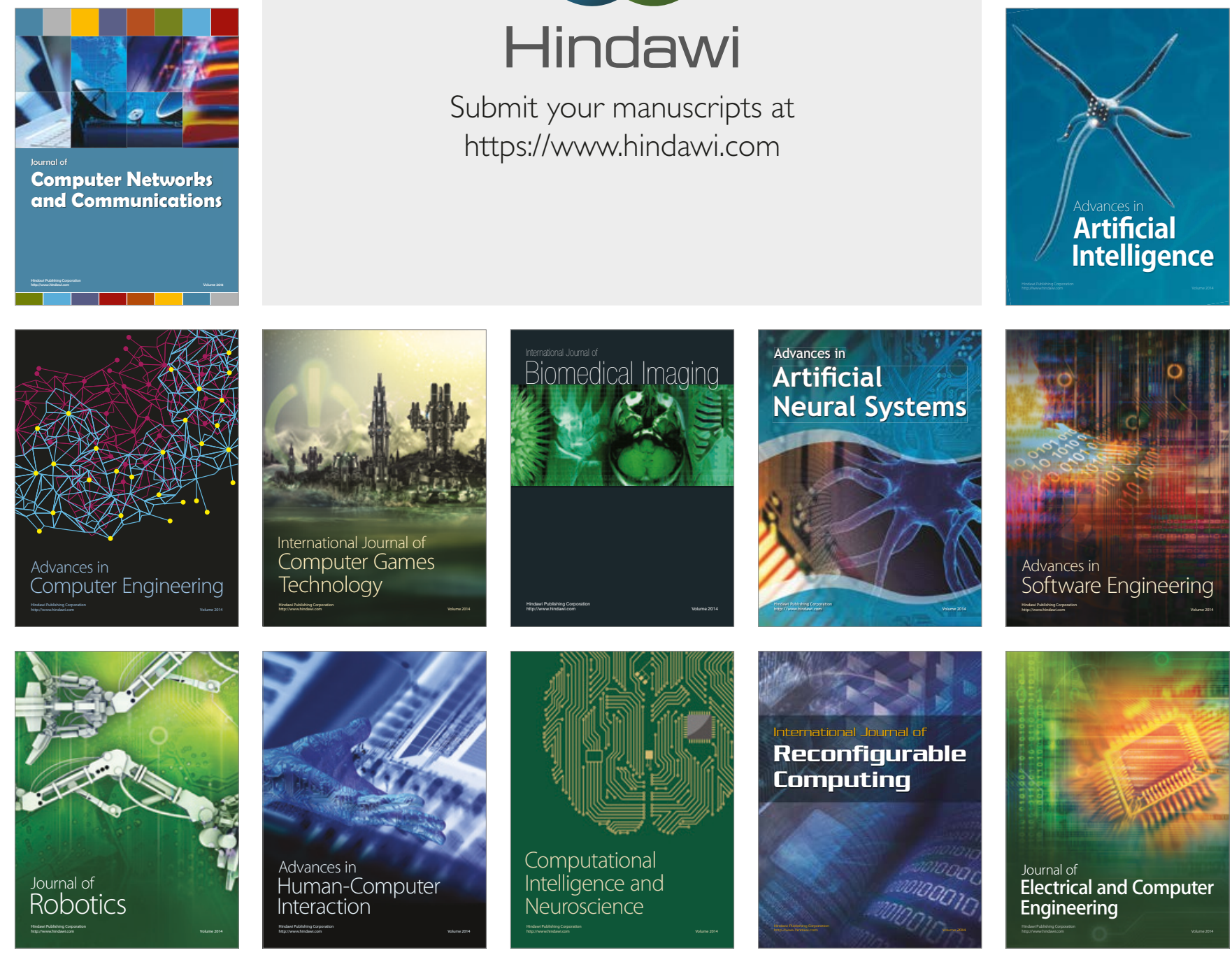